\title{
Is the resistance of gemcitabine for pancreatic cancer settled only by overexpression of deoxycytidine kinase?
}

\author{
NAOTAKE FUNAMIZU ${ }^{1,2}$, AIKOU OKAMOTO ${ }^{4}$, YUKO KAMATA ${ }^{3}$, TAKEYUKI MISAWA $^{1}$, \\ TADASHI UWAGAWA ${ }^{1}$, TAKESHI GOCHO ${ }^{1}$, KATSUHIKO YANAGA ${ }^{1}$ and YOSHINOBU MANOME ${ }^{2}$ \\ ${ }^{1}$ Department of Surgery, ${ }^{2}$ Department of Molecular Cell Biology and ${ }^{3}$ Oncology, Institute of DNA Medicine, \\ ${ }^{4}$ Department of Gynecology, School of Medicine, The Jikei University School of Medicine, \\ 3-25-8 Nishi-shinbashi, Minato-ku, Tokyo 105-8461, Japan
}

Received August 27, 2009; Accepted October 29, 2009

DOI: $10.3892 /$ or_00000657

\begin{abstract}
The prognosis of pancreatic cancer remains poor, and the standard first-line chemotherapy with gemcitabine (GEM) has a response rate of less than $20 \%$. Since expression of deoxycytidine kinase (dCK) seems important for improvement of GEM sensitivity, overexpression of dCK was investigated using pancreatic cancer cell lines (Panc-1, MIAPaCa-2 and $\mathrm{BxPC}-3$ ). dCK gene was introduced into the cell lines by retrovirus and changes in IC50 were examined. Sensitivity of two pancreatic cancer cell lines to GEM elevated dramatically in comparison with control cells, but change of sensitivity remained at 1.8 times in $\mathrm{BxPC}-3$. Since addition of tetrahydro uridine (THU), an inhibitor of deoxycytidine deaminase (CDA), increased the sensitivity 54-fold, overexpression of CDA seems to be the mechanism for improvement of the sensitivity. In conclusion, dCK is a key enzyme of GEM, but resistance of GEM is not improved in all pancreatic cancer cells by overexpression of $\mathrm{dCK}$. Combination treatment based on expression of GEM metabolism-related gene may become an effective therapy in the future.
\end{abstract}

\section{Introduction}

The prognosis of pancreatic cancer remains poor, for which surgery remains the only potentially curative treatment. This poor prognosis relates to the advanced disease stage at the time of diagnosis and to its profound resistance to existing therapies. Gemcitabine (GEM) is a cytotoxic pyrimidine

Correspondence to: Dr Naotake Funamizu, Department of Surgery, The Jikei University School of Medicine, 3-25-8 Nishishinbashi, Minato-ku, Tokyo 105-8461, Japan

E-mail: funamizu@jikei.ac.jp

Abbreviations: GEM, gemcitabine; dCK, deoxycytidine kinase; THU, tetra-hydro uridine; CDA, deoxycytidine deaminase

Key words: gemcitabine, pancreatic cancer, deoxycytidine kinase, deoxycytidine deaminase, tetra-hydro uridine deoxynucleoside analogue, with activity against several solid tumors such as cancers of the pancreas, lung, breast and ovary. GEM is transported into the cell mostly by human equilibrative and concentrative nucleoside transporters (hENT and hCNT, respectively). Cells deficient in hENT1 are highly resistant to GEM (1), while hCNT1 transfection increases GEM sensitivity in pancreatic cancer cell lines (2). This drug is activated by deoxycytidine kinase (dCK), and phosphorylates a monophosphate, diphosphate and triphosphate ( $\mathrm{dFdCTP})$. dCK is the rate-limiting enzyme in the transformation of nucleoside analogs, and the increase in $\mathrm{dCK}$ activity may improve the efficacy of $\operatorname{GEM}(3,4)$. dFdCTP is mainly incorporated into DNA leading to masked chain termination. In addition, its active metabolite can inhibit ribonucleotide reductase (RR), resulting in a decrease in deoxynucleoside triphosphate (dNTP) pools that are required for DNA repair and synthesis as well as inhibition of DNA polymerase. GEM is inactivated by deamination and catalyzed by deoxycytidine deaminase (CDA) (Fig. 1). Mechanism of intrinsic resistance to GEM in carcinomas remains unclear although that of acquired resistance to the drug in pancreatic carcinoma has been intensively studied. Some of these studies have disclosed a decrease in dCK activity (2), increased activity of CDA (5), and increased RR activity $(6,7)$. The aim of this study was to prove whether overexpression of dCK by retrovirus vector improves the therapeutic efficacy of GEM for pancreatic cancer.

\section{Materials and methods}

Reagent and chemicals. GEM (difluorodeoxycytidine, $\mathrm{dFdC}$ ) was a generous gift of Eli Lilly (Indianapolis, IN, USA). Drugs were dissolved in sterile distilled water and diluted in culture medium immediately before use. Tetrahydrouridine (THU) was purchased from Calbiochem (La Jolla, CA, USA) as a sterile white powder in glass vials and stored at $-20^{\circ} \mathrm{C}$.

Retroviral vector. Retroviral vector and a wild-type dCK cDNA have been described previously (8). The retroviral vector pMV-7 and pMV-7-dCK are transcribed from the murine Molony leukemia virus long terminal repeat (LTR). The neomycin resistance gene is transcribed from a thymidine kinase promoter (tk). 


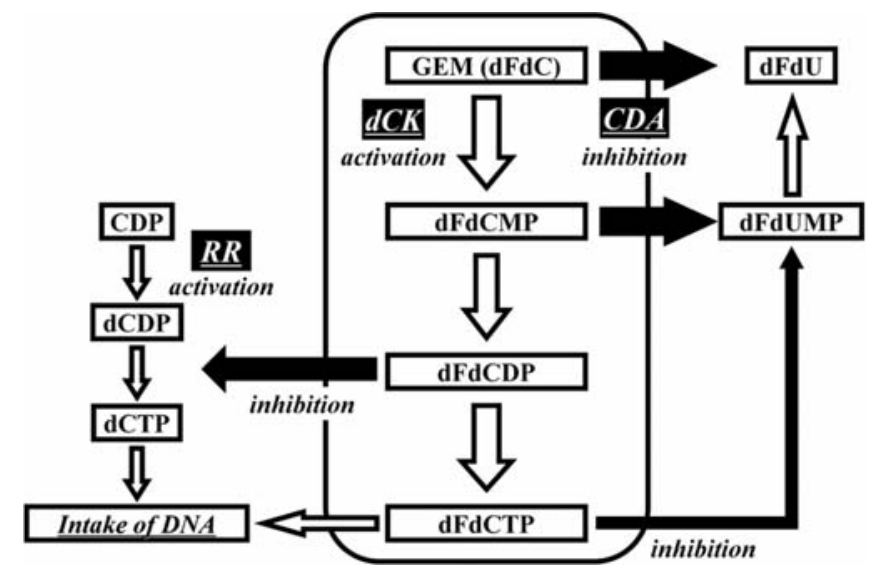

Figure 1. Metabolic pathway of gemcitabine (GEM). GEM is taken in DNA through phosphorylation by dCK. On the contrary, CDA inactivates GEM by deamination.

Cell lines and retroviral infection. The following human pancreatic adenocarcinoma cell lines were used in this study: Panc-1, MIAPaCA-2 and BxPC-3, which were obtained from the American Type Culture Collection (ATCC, Manassas, VA, USA). The amphotropic PA317 retrovirus packaging cell line was purchased from the same manufacturer. Panc-1 and PA317 cells were grown in DMEM medium supplemented with $10 \%$ fetal bovine serum (FBS), and MIAPaCa-2 was grown in DMEM medium supplemented with $15 \%$ FBS with antibiotics $(100 \mathrm{U} / \mathrm{ml}$ penicillin, $100 \mu \mathrm{g} / \mathrm{ml}$ streptomycin and $0.25 \mu \mathrm{g} / \mathrm{ml}$ amphotericin B). BxPC-3 cells were cultivated in RPMI-1640 in the presence of $10 \%$ FBS and the same antibiotics. All cell lines were routinely maintained as monolayer cultures at $37^{\circ} \mathrm{C}$ and $5 \% \mathrm{CO}_{2}$ atmosphere. Cells were harvested with $0.05 \%$ EDTA and $0.5 \%$ trypsin.

Construction of dCK expressing retroviral vectors and stable transfectants. Three different cell lines were transduced by the pMV-7-dCK or control pMV-7 using viral supernatant from the respective PA317 producer cell lines. The tissue culture medium was filtered, and the viral supernatant was added in the pancreatic cancer cell lines. The cells were incubated for $48 \mathrm{~h}$, and then polyclonal selection of stable cell lines integrated by retrovirus was performed by adding $100 \mu \mathrm{g} / \mathrm{ml}$ geneticin sulfate (Gibco BRL, Gaithersburg, MD, USA) to the culture media and cultured for 4 weeks. Respective stable cell lines (Panc-MV-7, Panc-dCK, MIAMV-7, MIA-dCK, BxPC-MV-7, BxPC-dCK) tranduced by

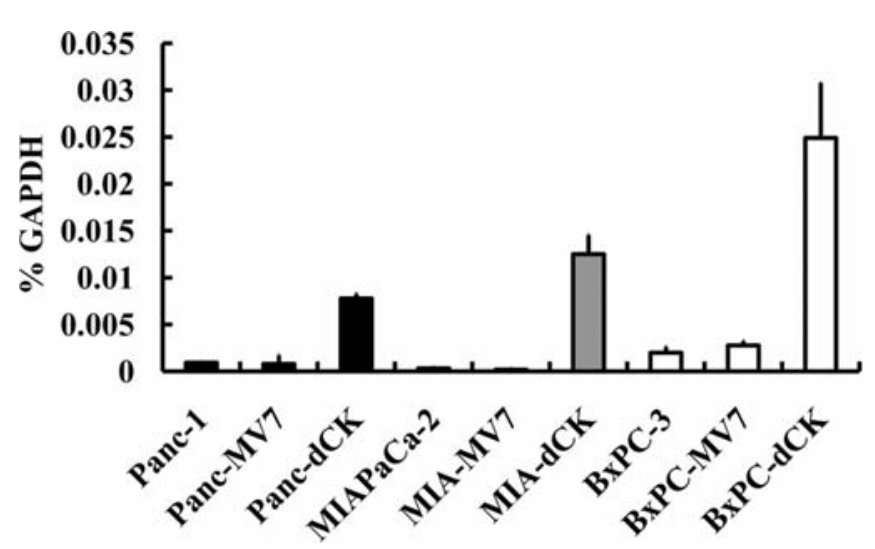

Figure 2. Expression of dCK mRNA of cell lines transfected by a retrovirus vector.

pMV-7 or pMV-7-dCK were characterized for dCK expression by real-time PCR analysis.

RNA preparation and real-time polymerase chain reaction (real-time PCR). The isolation of RNA was performed with RNAzol as previously described (9). Cell pellets were suspended in an aliquot of $1 \mathrm{ml}$ of RNAzol per $3 \mathrm{~cm}$ dish. Six micrograms of the isolated RNA was used for reverse transcription into cDNA (GE Health Care, Buckinghamshire, UK). Random hexamers were used as primers according to the manufacturer's instructions. After incubation at $65^{\circ} \mathrm{C}$ for $10 \mathrm{~min}$ to remove secondary structures, samples were quickly cooled down on ice and annealing of the hexamers also took place on ice. The reaction was terminated by heating at $37^{\circ} \mathrm{C}$ for $60 \mathrm{~min}$. cDNA samples were diluted and stored at $-20^{\circ} \mathrm{C}$ until further use. The design of the dCK primers is shown in Table I, which were selected requiring an optimal annealing temperature of $58^{\circ} \mathrm{C}$, absence of hairpins and no predictable stable primer-dimmer formations. Real-time PCR was performed in triplicates using the ABI PRISM 7300 sequence detection system (Applied Biosystems, Foster City, CA, USA) with the SYBR Green PCR Master mix (Applied Biosystems, Warrington, UK). The PCR conditions were as follows: one cycle at $95^{\circ} \mathrm{C}$ for $10 \mathrm{~min}$ followed by 40 cycles at $95^{\circ} \mathrm{C}$ for $15 \mathrm{sec}$ and $58^{\circ} \mathrm{C}$ for $30 \mathrm{sec}$, and $72^{\circ} \mathrm{C}$ for $30 \mathrm{sec}$. After amplification, dissociation curves were drawn to ensure that a single PCR product had been amplified. Products were also analyzed by gel electrophoresis and sequencing on first

Table I. Blue print of primer used with RT-PCR.

\begin{tabular}{lllr}
\hline Gene & \multicolumn{1}{c}{ Forward primer } & Reverse primer & Conditions \\
\hline dCK & ggaagtggttcctgaacctgttg & ctctgcatctttgagcttgcc & $58^{\circ} \mathrm{C} 33$ cycle \\
CDA & gaagcgtcctgcctgca & ctggaccgtcatgacaatatacg & $58^{\circ} \mathrm{C} 33$ cycle \\
GAPDH & gaaggtgaaggtcggagtc & gaagatggtgatgggatttc & $58^{\circ} \mathrm{C} 28$ cycle \\
\hline
\end{tabular}


A

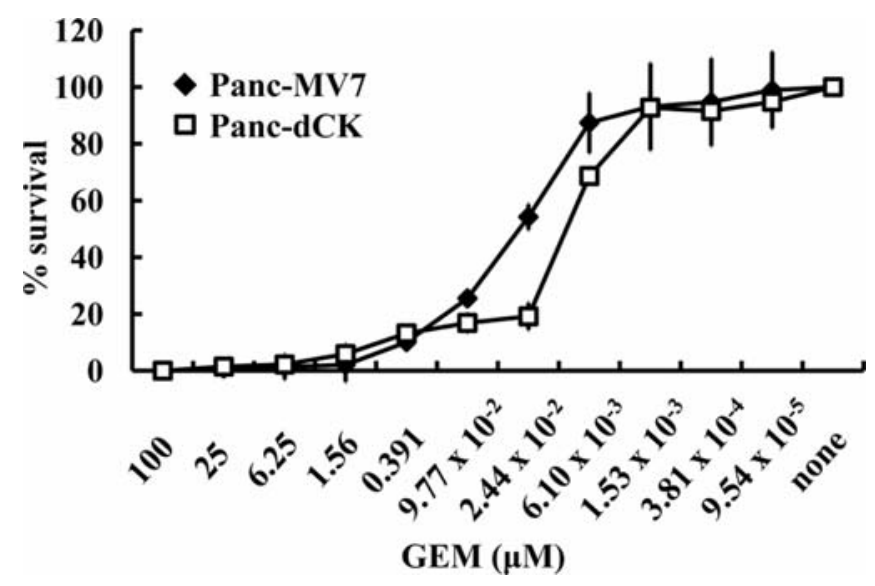

B

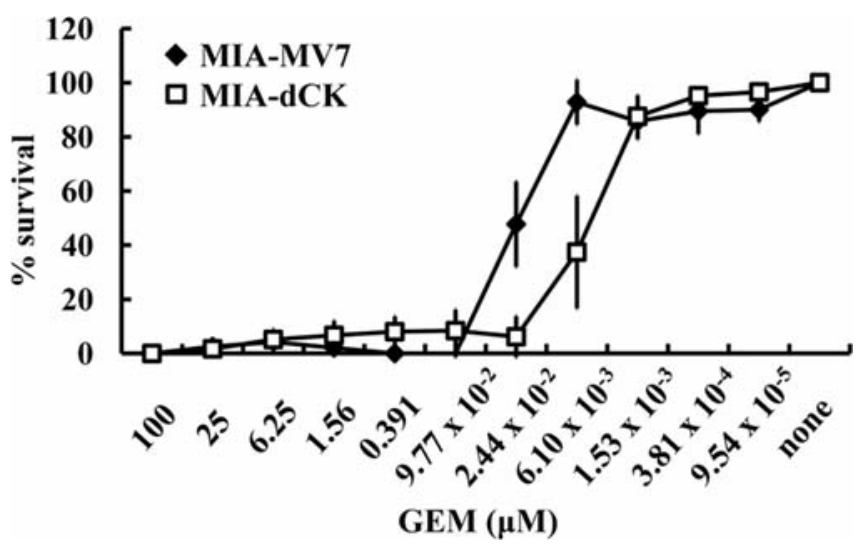

C

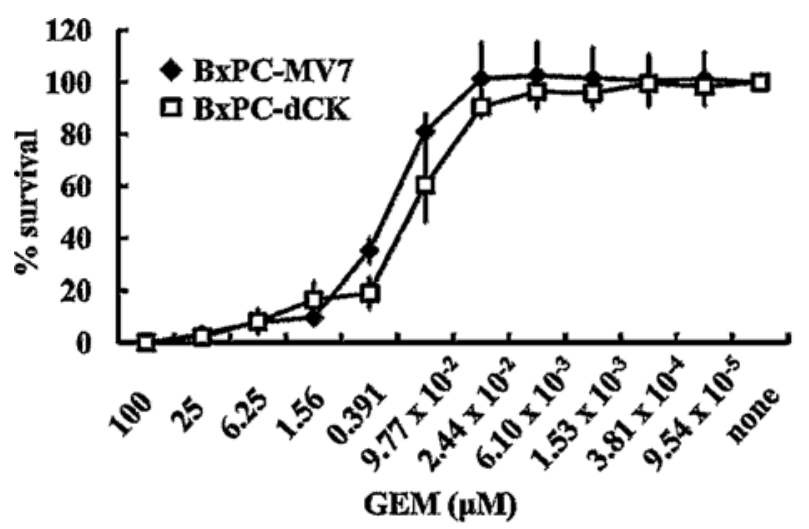

Figure 3. Sensitivity for GEM increased by transduction of dCK gene. Sensitivity of GEM by transduction of dCK gene increased in each cell line, 3.19 times for Panc-1, 5.18 times for MIAPaCa-2 and 1.85 times for BxPC-3.

primer pair usage to ensure that the correct gene fragment was amplified. All the reactions were performed in triplicates, and the standard method was used for the quantification of the expression for each segment, by use of $G A P D H$ as a normalization control gene.

Cytotoxic assay. Cells were seeded in 96-well sterile plastic plates (Costar) at 5000 cells/well. After $12 \mathrm{~h}$, cells were treated with stepwise 2-fold dilution of GEM $(\geq 100 \mu \mathrm{M})$ to assess cell viability, and incubated at $37^{\circ} \mathrm{C}$ for $96 \mathrm{~h}$. The

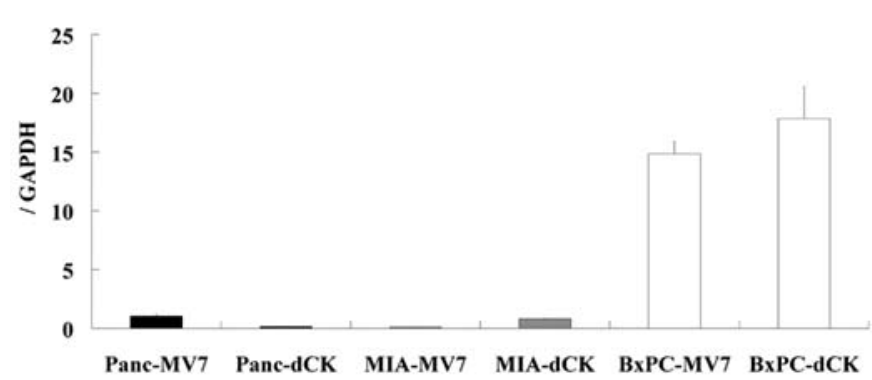

Figure 4. Expression of CDA mRNA level was examined by real-time PCR. BxPC-3 cell lines showed poor sensitivity to GEM.

cells were fixed at $25 \%$ glutaraldehide after drug exposure for $30 \mathrm{~min}$ at room temperature and then viable cells were stained with $200 \mu 1$ of $0.05 \%$ methylene blue for $20 \mathrm{~min}$ to evaluate the cytotoxicity of GEM. The dye was eluted with $0.33 \mathrm{M} \mathrm{HCl}$ for $20 \mathrm{~min}$ with agitation. Absorbance was measured in a microplate reader (model 3550, Bio-Rad, Tokyo, Japan) at $598 \mathrm{~nm}$. Mean values were calculated from three different wells in triplicates. Chemosensitivity was evaluated by IC50.

\section{Results}

dCK stable cell lines (Panc-dCK, MIA-dCK and BxPC-dCK) and control cell lines (Panc-MV-7, MIA-MV-7 and BxPCMV-7) were established to estimate a change of GEM sensitivity by overexpression of dCK. pMV-7 or pMV-dCK vector was transfected in retroviral packaging cells (PA317). After two days, the supernatant was collected and cells were precipitated with centrifuge at $3000 \mathrm{rpm}$ and assumed as the stock of virus liquid. Cell lines infected with retrovirus were selected by G418 for 3 weeks. Expression level of dCK of each cell line was measured by real-time PCR. The expression level of Panc-dCK, MIA-dCK and BxPC-dCK were 9.02, 9.69 and 71.4 times, respectively, higher than their controls (Panc-MV7, MIA-MV7 and BxPC-MV7) respectively (Fig. 2). IC50 of control cell lines (Panc-MV7, MIAMV7 and BxPC-MV7) to GEM was 29.77 $\pm 4.11,23.26 \pm 6.87$ and $256.12 \pm 17.4 \mathrm{nM}$ after $96 \mathrm{~h}$ of exposure, respectively. The sensitivity of BxPC-MV-7 to GEM was $>8$ times lower than the other cell lines (Panc-MV-7, MIA-MV-7 cells). Similarly GEM was administered with a dCK induction cell to examine change in IC50, transducing of dCK gene increased the sensitivity to GEM by $3.19,5.18$ and 1.85 times, respectively (Fig. 3). Though the expression of dCK increased $>70$ times in BxPC-dCK cells, IC50 did not change much in comparison with other cell lines. mRNA expression of metabolism-related gene was examined by RT-PCR. Expression of CDA, dCMPDA, TK2, 5-NT, p53, hENT-1, RRM1 and RRM2 was confirmed with all cell lines. The expression of other mRNA was almost at the same level (data not shown), but only CDA mRNA level showed significant difference (Fig. 4). Expression of CDA developed $>14$ times in BxPC$\mathrm{dCK}$ in comparison with Panc-dCK or MIA-dCK. When THU, an inhibitor of CDA with GEM, was given, only the sensitivity of BxPC-dCK cell line increased by as high as 
A

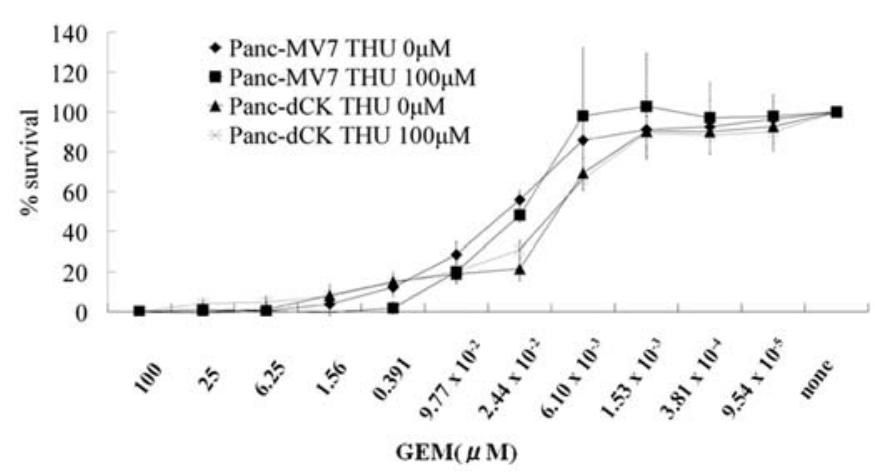

B

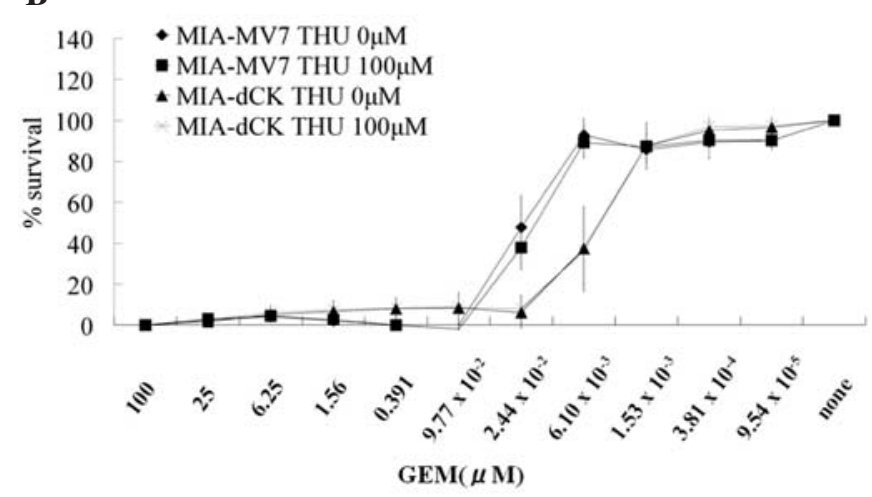

C

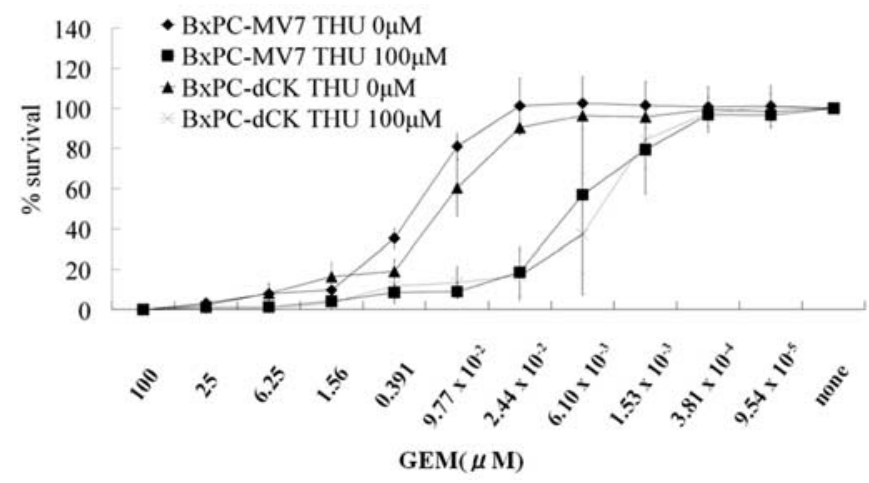

Figure 5. In the cytotoxic assay, the addition of THU, an inhibitor of CDA, increased the sensitivity of BxPC-3 to GEM.

54 times (Fig. 5). Panc-dCK, MIA-dCK-cell lines showed minimal change in IC50.

\section{Discussion}

The effectivity of gene therapy by single gene induction or anticancer drug is limited for treatment of pancreatic cancer, because the chemosensitibity to GEM differs depending on genetic expression of the pancreatic cancer. The combination treatment that matched with genetic expression is required to treat pancreatic cancer of variable characteristics. Our study suggested that the combination of $\mathrm{dCK}$ and THU may be an effective treatment for pancreatic cancer.

Various studies have reported on mechanism of GEMresistance in pancreatic cancer, especially on dCK $(3,4,10-12)$, CDA $(5,13,14), \operatorname{RR}(6,7,15)$ and hENT $(1,2,16-20)$. dCK gene, a key enzyme of GEM metabolism, was transduced by retrovirus into three cell lines, Panc-1, MIAPaCa-2 and BxPC-3. Expression level of dCK mRNA in the cell lines transduced with dCK gene were examined and compared with control cells by RT-PCR. The sensitivity to GEM was not in proportion to expression of $\mathrm{dCK}$ in our study. Therefore, overexpression of $\mathrm{dCK}$ was not effective in all pancreatic cancer cell lines.

As to the cause, overexpression of CDA was suggested, since CDA metabolizes GEM rapidly to its inactive metabolite, and CDA is known to be associated with GEM resistance.

$\mathrm{dCK}$ is a key enzyme, but is not necessarily effective in all pancreatic cancer cell lines. Treatment in accordance with the characteristics of the pancreatic cancer cells is needed. Many studies have reported the effectiveness of combination treatment of anticancer drug and inhibitor or gene transduction (22-32). There is no treatment that is effective for all carcinomas with various characteristics. These regimens may be more effective if the regimen is chosen depending on gene expression of pancreatic cancer.

In conclusion, THU is effective for pancreatic cancer when CDA is expressed at high level, and GEM sensitivity increased by transducing the dCK gene. Therefore, combination treatment depending on gene expression could be useful.

\section{References}

1. Spratlin J, Sangha R, Glubrecht D, Dabbagh L, Young JD, Dumontet C, Cass C, Lai R and Mackey JR: The absence of human equilibrative nucleoside transporter 1 is associated with reduced survival in patients with gemcitabine treated pancreas adenocarcinoma. Clin Cancer Res 10: 6956-6961, 2004.

2. Garcia-Manteiga J, Molina-Arcas M, Casado FJ, Mazo A and Pastor-Anglada M: Nucleoside transporter profiles in human pancreatic cancer cells: role of hCNT1 in 2',2,-difluorodeoxycytidine-induced cytotoxicity. Clin Cancer Res 9: 5000-5008, 2003.

3. Kroep JR, Loves WJ, van der Wilt CL, et al: Pretreatment deoxycytidine kinase levels predict in vivo gemcitabine sensitivity. Mol Cancer Ther 1: 371-376, 2002.

4. Blackstock AW, Lightfoot H, Case LD, et al: Tumor uptake and elimination of 2',2,-difluorodeoxy-cytidine (gemcitabine) after deoxycytidine kinase gene transfer correlation with in vivo tumor response. Clin Cancer Res 7: 3263-3268, 2001.

5. Neff T and Blau CA: Forced expression of cytidine deaminase confers resistance to cytosine arabinoside and gemcitabine. Exp Hematol 24: 1340-1346, 1996.

6. Bergman AM, Eijk PP, Ruiz van Haperen VW, Smid K, Veerman G, Hubeek I, van den Ijssel P, Ylstra B and Peters GJ: In vivo induction of resistance to gemcitabine results in increased expression of ribonucleotide reductase subunit M1 as the major determinant. Cancer Res 65: 9510-9516, 2005.

7. Nakahira S, Nakamori S, Tsujie M, et al: Involvement of ribonucleotide reductase M1 subunit overexpression in gemcitabine resistance of human pancreatic cancer. Int J Cancer 120: 1355-1363, 2003 .

8. Manome Y, Wen PY, Dong Y, Tanaka T, Mitchell BS, Kufe DW and Fine HA: Viral vector transduction of the human deoxycytidine kinase cDNA sensitizes glioma cells to the cytotoxic effects of cytosine arabinoside in vitro and in vivo. Nat Med 2: 567-573, 1996.

9. Chomczynski P and Sacchi N: Single-step method of RNA isolation by acid guanidium thiocyanate-phenol-chloroform extraction. Anal Biochem 162: 156-159, 1987.

10. Galmarini CM, Clarke ML, Jordheim L, Santos CL, Cros E, Mackey JR and Dumontet C: Resistance to gemcitabine in a human follicular lymphoma cell line is due to partial deletion of the deoxycytidine kinase gene. BMC Pharmacol 24: 4-8, 2004. 
11. Achiwa H, Oguri T, Sato S, Maeda H, Niimi T and Ueda R: Determinants of sensitivity and resistance to gemcitabine: the roles of human equilibrative nucleoside transporter 1 and deoxycytidine kinase in non-small cell lung cancer. Cancer Sci 95: 753-757, 2004

12. Jordheim LP, Galmarini CM and Dumontet C: Gemcitabine resistance due to deoxycytidine kinase deficiency can be reverted by fruitfly deoxynucleoside kinase, DmdNK, in human uterine sarcoma cells. Cancer Chemother Pharmacol 58: 547-554, 2006.

13. Bengala C, Guarneri V, Giovannetti E, et al: Prolonged fixed dose rate infusion of gemcitabine with autologous haemopoietic support in advanced pancreatic adenocarcinoma. Br J Cancer 93: 35-40, 2005.

14. Bouffard DY, Laliberté J and Momparler RL: Kinetic studies on 2',2'-difluorodeoxycytidine (Gemcitabine) with purified human deoxycytidine kinase and cytidine deaminase. Biochem Pharmacol 45: 1857-1861, 1993.

15. Smid K, Bergman AM, Eijk PP, et al: Micro-array analysis of resistance for gemcitabine results in increased expression of ribonucleotide reductase subunits. Nucleosides Nucleotides Nucleic Acids 25: 1001-1007, 2006.

16. Mori R, Ishikawa T and Ichikawa Y: Human equilibrative nucleoside transporter 1 is associated with the chemosensitivity of gemcitabine in human pancreatic adenocarcinoma and biliary tract carcinoma cells. Oncol Rep 17: 1201-1205, 2007.

17. Giovannetti E, Del Tacca M, Mey V, et al: Transcription analysis of human equilibrative nucleoside transporter-1 predicts survival in pancreas cancer patients treated with gemcitabine. Cancer Res 66: 3928-3935, 2006.

18. Hubeek I, Stam RW, Peters GJ, et al: The human equilibrative nucleoside transporter 1 mediates in vitro cytarabine sensitivity in childhood acute myeloid leukaemia. Br J Cancer 93: 1388-1394, 2005.

19. Damaraju VL, Damaraju S, Young JD, Baldwin SA, Mackey J, Sawyer MB and Cass CE: Nucleoside anticancer drugs: the role of nucleoside transporters in resistance to cancer chemotherapy. Oncogene 22: 7524-7536, 2003.

20. Mata JF, García-Manteiga JM, Lostao MP, et al: Role of the human concentrative nucleoside transporter (hCNT1) in the cytotoxic action of 5(Prime)-deoxy-5-fluorouridine, an active intermediate metabolite of capecitabine, a novel oral anticancer drug. Mol Pharmacol 59: 1542-1548, 2001.

21. Rauchwerger DR, Firby PS, Hedley DW and Moore MJ: Equilibrative-sensitive nucleoside transporter and its role in gemcitabine sensitivity. Cancer Res 60: 6075-6079, 2000.
22. Matthews DJ, Yakes FM, Chen J, et al: Pharmacological abrogation of S-phase checkpoint enhances the anti-tumor activity of gemcitabine in vivo. Cell Cycle 6: 104-110, 2007.

23. Mey V, Giovannetti E, De Braud F, et al: In vitro synergistic cytotoxicity of gemcitabine and pemetrexed and pharmacogenetic evaluation of response to gemcitabine in bladder cancer patients. Br J Cancer 95: 289-297, 2006.

24. Hoffmann D and Wildner O: Enhanced killing of pancreatic cancer cells by expression of fusogenic membrane glycoproteins in combination with chemotherapy. Mol Cancer Ther 5: 2013-2022, 2006

25. Bianco C, Giovannetti E, Ciardiello F, et al: Synergistic antitumor activity of ZD6474, an inhibitor of vascular endothelial growth factor receptor and epidermal growth factor receptor signaling, with gemcitabine and ionizing radiation against pancreatic cancer. Clin Cancer Res 12: 7099-7107, 2006.

26. Giovannetti E, Mey V, Nannizzi S, Pasqualetti G, Marini L, Del Tacca $\mathrm{M}$ and Danesi R: Cellular and pharmacogenetics foundation of synergistic interaction of pemetrexed and gemcitabine in human non-small cell lung cancer cells. Mol Pharmacol 68: 110-118, 2005.

27. Vernejoul F, Ghénassia L, Souque A, et al: Gene therapy based on gemcitabine chemosensitization suppresses pancreatic tumor growth. Mol Ther 14: 758-767, 2006.

28. Bocci G, Fioravanti A, Orlandi P, Bernardini N, Collecchi P, Del Tacca $M$ and Danesi R: Fluvastatin synergistically enhances the antiproliferative effect of gemcitabine in human pancreatic cancer MIAPaCa-2 cells. Br J Cancer 93: 319-330, 2005.

29. Yao B, He QM, Tian L, et al: Enhanced antitumor effect of the combination of tumstatin gene therapy and gemcitabine in murine models. Hum Gene Ther 16: 1075-1086, 2005.

30. Hamada S, Satoh K, Kimura K, Kanno A, Masamune A and Shimosegawa T: MSX2 overexpression inhibits gemcitabineinduced caspase-3 activity in pancreatic cancer cells. World J Gastroenterol 11: 6867-6870, 2005.

31. Duxbury MS, Ito H, Zinner MJ, Ashley SW and Whang EE: RNA interference targeting the M2 subunit of ribonucleotide reductase enhances pancreatic adenocarcinoma chemosensitivity to gemcitabine. Oncogene 23: 1539-1548, 2004.

32. Kindler HL: The pemetrexed/gemcitabine combination in pancreatic cancer. Cancer 95: 928-932, 2002. 